\title{
Nonlinear dynamics of a parametrically driven sine-Gordon system
}

\author{
Grønbech-Jensen, Niels; Kivshar, Yuri S.; Samuelsen, Mogens Rugholm
}

Published in:

Physical Review B

Link to article, DOI:

10.1103/PhysRevB.47.5013

Publication date:

1993

Document Version

Publisher's PDF, also known as Version of record

Link back to DTU Orbit

Citation (APA):

Grønbech-Jensen, N., Kivshar, Y. S., \& Samuelsen, M. R. (1993). Nonlinear dynamics of a parametrically driven sine-Gordon system. Physical Review B, 47(9), 5013-5021. https://doi.org/10.1103/PhysRevB.47.5013

\section{General rights}

Copyright and moral rights for the publications made accessible in the public portal are retained by the authors and/or other copyright owners and it is a condition of accessing publications that users recognise and abide by the legal requirements associated with these rights.

- Users may download and print one copy of any publication from the public portal for the purpose of private study or research.

- You may not further distribute the material or use it for any profit-making activity or commercial gain

- You may freely distribute the URL identifying the publication in the public portal

If you believe that this document breaches copyright please contact us providing details, and we will remove access to the work immediately and investigate your claim 


\title{
Nonlinear dynamics of a parametrically driven sine-Gordon system
}

\author{
Niels Grønbech-Jensen \\ Department of Applied Physics, Stanford University, Stanford, California 94305 \\ Yuri S. Kivshar* \\ Departamento de Fisica Teorica I, Facultad de Ciencias Fisicas, Universidad Complutense, 28040 Madrid, Spain \\ Mogens R. Samuelsen \\ Physics Laboratory I, The Technical University of Denmark, 2800 Lyngby, Denmark \\ (Received 6 February 1992; revised manuscript received 21 October 1992)
}

\begin{abstract}
We consider a sine-Gordon system, driven by an ac parametric force in the presence of loss. It is demonstrated that a breather can be maintained in a steady state at half of the external frequency. In the small-amplitude limit the effect is described by an effective nonlinear Schrödinger equation. For an arbitrary frequency of the applied field the threshold field for the breather stabilization is determined by a perturbation method and it is compared to direct numerical simulations. We also analyze the variation of the breather parameters in the stationary regime and calculate the frequency of such a modulation for a general type of driving force.
\end{abstract}

\section{INTRODUCTION}

The sine-Gordon (SG) model is known to describe many physical objects in a one-dimensional approximation, mostly, in solid-state physics, for example, flux propagation in long Josephson junctions, dislocations in solids, nonlinear spin waves in superfluid phases of ${ }^{3} \mathrm{He}$, ferromagnetic, or antiferromagnetic systems, etc. The simplest localized (soliton) solutions of the model are divided into two different classes, namely, kinks and breathers. The kink solutions describe motion of topological excitations, for example, flux quanta (fluxons) in long Josephson transmission lines or various domain walls in magnetic systems (see, e.g., the review paper, Ref. 1 and references therein). The kink connects two nearest ground states of the system, say $u=0$ and $u=2 \pi$ when the field variable $u$ is an angle, so that this topological object may be called a " $2 \pi$ kink." In real physical systems when dissipative losses are included, the kinks may exist in the form of static stable configurations, and they will move under an external force. ${ }^{1}$

The other type of soliton solutions, the so-called "breathers," may be considered as dynamical bound states of kink-antikink pairs, and they are nonlinear oscillating states. Under the influence of dissipative forces and constant fields, the breather excitations usually decay into either a kink-antikink pair or small-amplitude (linear) waves eventually decaying because of dissipation.

In a number of physically important systems, an applied external field is periodic in time. When an ac field is applied to the SG system, it can compensate for the dissipative losses and maintain a stationary breather. In the small-amplitude limit, when the SG system is described by an effective nonlinear Schrödinger (NLS) equation, the problem was considered by Kaup and Newell, ${ }^{2}$ who found a phase-locked soliton by means of perturbation theory. A more detailed investigation reveals complicat- ed and stochastic dynamics of the ac driven SG system with loss. ${ }^{3-10}$ The main physically important result is the following: a small-amplitude ac driving force may compensate for dissipative losses so that a SG breather can be maintained as a stabilized phase-locked object.

In other physically important systems, e.g., magnetic $^{11-12}$ or long Josephson junctions ${ }^{15-17}$ the applied periodic force acts parametrically, i.e., it varies the parameters of the model. As was demonstrated in our previous paper, ${ }^{13}$ a parametric force may stabilize a breather and the threshold amplitude of the force may be calculated analytically.

It is the purpose of this paper to give a detailed analysis of the SG dynamics under the presence of external parametric forces of different kinds. For such a parametric force we present an extended analysis of the breather dynamics in the phase-locked regime. In particular, we analyze analytically and numerically a modulation of the breather frequency around its steady-state value.

The paper is organized as follows: in Sec. II we describe our model and discuss some of its applications. Section III is devoted to the stabilization of the SG breather dynamics in the presence of a LF parametric force. In Sec. IV we analyze the modulation of the phase-locked breather frequency. In this section it is also demonstrated that a general expression for the modulation frequency of the breather frequency can be found, regardless of the origin of the drive. In all problems presented in this paper, the analytical results are in excellent agreement with direct numerical simulations of the corresponding nonlinear effects. Section V concludes the paper.

\section{THE MODEL}

The model we deal with in this paper is described by the perturbed SG equation 


$$
\phi_{x x}-\phi_{t t}-\sin \phi=\alpha \phi_{t}-\varepsilon_{n} \sin \omega t \sin (\phi / n),
$$

where $\alpha$ is the loss parameter, $\varepsilon_{n}$ is the amplitude of the external parametric force, $\omega$ is its frequency, and $n$ is a rational number. Equation (1) arises, for example, as an effective equation of motion for the magnetization vector in magnetic systems, $\phi$ being an angle describing its orientation in a selected plane. For definiteness, a onedimensional easy-plane ferromagnetic like $\mathrm{CsNiF}_{3}$ or $T M M C$ is described by the well-known Landau-Lifshitz equation in which the dimensionless form may be written as the following:

$\mathbf{M}_{t}=\mathbf{M} \times \mathbf{M}_{x x}-\beta(\mathbf{M} \cdot \mathbf{e})(\mathbf{M} \times \mathbf{e})+\mathbf{M} \times \mathbf{H}-\alpha \mathbf{M} \times \mathbf{M}_{t}$,

where $\mathbf{M}$ is the unit (normalized) magnetization vector, $\beta$ $(\beta>0)$ is the easy-plane anisotropy constant, $\mathbf{e}=(1,0,0)$, and $\alpha$ is a relaxation constant. If we consider the magnetic field in the easy plane, $\mathbf{H}=[H(t), 0,0]$, when in the limit $\beta \gg\langle H\rangle,\langle H\rangle$ being the field averaged over the period of oscillation, we may derive the following SG equation, $\phi$ being the easy-plane angle, from Eq. (2):

$$
\phi_{x x}-\beta^{-1} \phi_{t t}-H(t) \sin \phi=\alpha \phi_{t},
$$

which is, after an appropriate rescaling, identical to Eq. (1) for $n=1 .^{18}$

Another example is the model of a weak two-sublattice ferromagnetic (antiferromagnetic) system. ${ }^{19}$ In that case the main nonlinearity $(\sim \sin \phi)$ is caused by anisotropy (unlike the previous example), and the parameter $\varepsilon_{2}(n=2)$ is proportional to the amplitude of the external (variable) magnetic field. Other examples of physical realizations for the system (1) may be found, e.g., in Ref. 1. However, we would like to mention additionally that recently a few papers have appeared ${ }^{15-17}$ in which the possibility of parametric excitations in Josephson junctions is discussed. For the latter case, such a parametric influence may change the critical current of the junction that may be modeled by Eq. (1) for $n=1$.

Without perturbations (i.e., $\varepsilon_{n}=\alpha=0$ ), Eq. (1) has two different types of soliton solutions. The kink is given by the expression

$$
\phi_{k}(x, t)=4 \tan ^{-1} e^{z}, \quad z= \pm \frac{x-v t}{\sqrt{1-v^{2}}}
$$

where the sign "+" or "-" corresponds to the kink polarity (kink or antikink).

The breather (at rest) has the form

$\phi_{\mathrm{br}}(x, t)=4 \tan ^{-1}\left(\frac{\sqrt{1-\omega_{\mathrm{br}}^{2}}}{\omega_{\mathrm{br}}} \frac{\sin \left(\omega_{\mathrm{br}} t+\theta\right)}{\cosh \left(x \sqrt{1-\omega_{\mathrm{br}}^{2}}\right)}\right)$,

$\omega_{\text {br }}$ being the breather frequency, $0<\omega_{\mathrm{br}}<1$, and $\theta$ is an arbitrary initial phase. The moving breather may be obtained from Eq. (4) by a Lorentz transformation.

\section{BREATHER STABILIZATION}

If the breather is maintained in the steady-state regime, all the terms in Eq. (1), except for the driving term, contain odd harmonics of $\omega_{\mathrm{br}}$. In order to support the solution, the driving term must therefore also contain odd harmonics of $\omega_{\mathrm{br}}$. To fulfill that we have to consider

$$
\omega=2 \omega_{\mathrm{br}},
$$

i.e., the condition of a parametric resonance.

In this section we will present our result for a generalized parametrically driven SG model,

$\phi_{x x}-\phi_{t t}-\sin \phi=\alpha \phi_{t}-\beta \phi_{x x t}-\varepsilon_{n}^{(1)} \sin \frac{\phi}{n} \sin \left(\omega t+\theta_{n}^{(1)}\right)-\varepsilon^{(2)} \phi_{t} \sin \left(\omega t+\theta^{(2)}\right)-\varepsilon^{(3)} \phi_{t t} \sin \left(\omega t+\theta^{(3)}\right)-\varepsilon^{(4)} \phi_{x x} \sin \left(\omega t+\theta^{(4)}\right)$

In the Introduction we have mentioned some physical applications of Eq. (6) at $\varepsilon_{n}^{(1)} \neq 0$. Here we add that the generalized model above is more relevant for applications in the theory of long Josephson junctions and driven pendulums. For example, the term proportional to $\varepsilon^{(2)}$ has been studied both theoretically and experimentally. ${ }^{20}$ This has been done for systems without spatial extension. The experiments for studying this term were made by modulating the friction periodically in a mechanical pendulum. In this section we will generalize the analysis ${ }^{13}$ made on the parametric term $\varepsilon_{n}^{(1)}$ to cover the terms $\varepsilon^{(2)}$, $\varepsilon^{(3)}$, and $\varepsilon^{(4)}$ as well as the loss term $\sim \beta .^{21}$

\section{A. Small-amplitude limit}

In the small-amplitude limit, $1-\omega_{\mathrm{br}}^{2}<<1$, we may look for a solution in the form

$$
\phi(x, t)=A(x, t) e^{i t}+\text { c.c. }
$$

$A(x, t)$ being a slowly varying envelope. Substituting Eq. (5) into Eq. (6) yields for $\left|A_{t t}\right| \ll\left|A_{t}\right| \ll|A|$ the perturbed NLS equation ${ }^{22}$

$$
\begin{aligned}
2 i A_{t}-A_{x x}-\frac{1}{2}|A|^{2} A= & -i \alpha A+\frac{\varepsilon_{n}^{(1)}}{2 i n}\left[A^{*} e^{-2 i \Omega t}\left(1-\frac{1}{2 n^{2}}|A|^{2}\right)+\frac{1}{6 n^{2}} A^{3} e^{2 i \Omega t}\right] \\
& -\frac{\varepsilon^{(2)}}{2} A^{*} e^{-2 i \Omega t}-\frac{\varepsilon^{(3)}}{2 i} A^{*} e^{-2 i \Omega t}+\frac{\varepsilon^{(4)}}{2 i} A_{x x}^{*} e^{-2 i \Omega t}
\end{aligned}
$$


where the asterisk means the complex conjugation. The effective driving frequency, $\Omega \equiv 1-\omega_{\mathrm{br}}$, is considered to be small. $A$ is, like all the terms in Eq. (8), oscillating with the frequency $-\Omega(\Omega<1)$, since $\phi$ oscillates with the frequency $\omega_{\mathrm{br}}$.

The unperturbed NLS equation $\left(\varepsilon_{n}=\alpha=0\right)$ has a solution in the slowly oscillating form

$$
A(x, t)=4 i \eta \frac{e^{-2 i \eta^{2} t}}{\cosh (2 \eta x)},
$$

$\eta$ being the parameter of the soliton. From the viewpoint of the SG system, $\eta=\frac{1}{2}\left(1-\omega_{\text {br }}^{2}\right)^{1 / 2}$ [cf. Eq. (4)]. To analyze the perturbation-induced dynamics of the oscillating NLS solution [Eq. (9)] we use a perturbation theory for solitons, ${ }^{1}$ considering the perturbative terms as small. According to this approach, we look for a perturbed solution in the form

$$
A(x, t)=4 i \eta(t) \frac{e^{-i \chi(t)}}{\cosh [2 \eta(t) x]}
$$

where $\eta(t)$ and $\dot{\chi}(t)$ are slowly varying functions.

Using the so-called adiabatic equations of the perturbation theory for solitons, ${ }^{1}$ we can obtain the equations for the time-dependent soliton parameters $\eta$ and $\psi$ :

$$
\begin{aligned}
\frac{d \eta}{d t}(1-\eta)= & -\frac{\alpha}{2} \eta+\frac{\varepsilon_{n}^{(1)}}{4 n}\left(1-\frac{32}{9 n^{2}} \eta^{2}\right) \eta \cos \psi \\
& +\frac{\varepsilon^{(2)}}{4} \eta \sin \psi-\frac{\varepsilon^{(3)}}{4} \eta \cos \psi-\frac{\varepsilon^{(4)}}{4} \eta^{3} \cos \psi
\end{aligned}
$$

$$
\begin{aligned}
\frac{d \psi}{d t}= & 4 \eta^{2}-2 \Omega-\frac{\varepsilon_{n}^{(1)}}{2 n}\left(1-\frac{64}{9 n^{2}} \eta^{2}\right) \sin \psi \\
& +\frac{\varepsilon^{(2)}}{2} \cos \psi+\frac{\varepsilon^{(3)}}{2} \sin \psi+\frac{\varepsilon^{(4)}}{3} \eta^{2} \cos \psi
\end{aligned}
$$

where $\psi(t)=2 \chi(t)-2 \Omega t$ denotes the phase of the soliton relative to the parametric drive. In a steady state, the amplitude $\eta$ and the phase $\psi$ equal their fix points $\eta=\eta_{*}$ and $\psi=\psi_{*}$. Treating each parametric force independently, we obtain from the lowest approximation in $\eta$ :

$$
\begin{aligned}
\psi_{*} & = \pm \arccos \left(2 \alpha n / \varepsilon_{n}^{(1)}\right), \\
\eta_{*}^{2} & =\frac{1}{4} \Omega \pm \frac{1}{4} \sqrt{\left(\varepsilon_{n}^{(1)} / 2 n\right)^{2}-\alpha^{2}} \\
& =\varepsilon_{n}^{(1)} / \alpha \geq 2 n .
\end{aligned}
$$

$$
\begin{aligned}
\psi_{*} & =\pi / 2 \pm\left[\pi / 2-\arcsin \left(2 \alpha / \varepsilon^{(2)}\right]\right. \\
\eta_{*}^{2} & =\frac{1}{4} \Omega \pm \frac{1}{4} \sqrt{\left(\varepsilon^{(2)} / 2\right)^{2}-\alpha^{2}} \\
& =\frac{\varepsilon^{(2)}}{\alpha} \geq 2 \\
\psi_{*} & =\pi \pm \arccos \left(2 \alpha / \varepsilon^{(3)}\right) \\
\eta_{*}^{2} & =\frac{1}{4} \Omega \pm \frac{1}{4} \sqrt{\left(\varepsilon^{(3)} / 2\right)^{2}-\alpha^{2}} \\
& =\varepsilon^{(3)} / \alpha \geq 2 .
\end{aligned}
$$

These solutions correspond to nonpropagating solitons whose amplitudes and phases are fixed but whose positions in space are arbitrary. Therefore the smallamplitude breather [Eq. (4)] considered as a NLS soliton is stabilized at one-half of the external frequency (as a parametrically phase-locked state). At the threshold the amplitude of the stabilized soliton is $\eta_{*}=\frac{1}{2} \sqrt{\Omega}$; however, for the soliton phase there is zero mismatch between the soliton and the phase of the parametric force. We conclude that the soliton can be maintained in an energy balance between the loss and a parametric force, but it is important to note that the parametrical term $\sim \varepsilon^{(4)}$ does not produce any phase-locked soliton state in this limit. The NLS regime fails to give us the threshold value of the amplitude of the applied drive for finite (but small) values of the detuning $\Omega$. Further, the threshold value of the external drive can be obtained only for $\omega_{b r}=1$, which is a somehow uninteresting point for the SG breather, since the amplitude of the breather is zero for this particular frequency. On the other hand, we can here differentiate stable from unstable fix points. As seen from Eqs. (13), (14), and (15), we have obtained the fix points in pairs. Using Eqs. (11) and (12) and making the stability analysis around $\left(\eta_{*}, \psi_{*}\right)$ we easily obtain that the stable fix points are given for $\varepsilon_{n}^{(1)}, \sin \psi_{*}>0 ; \varepsilon^{(2)}, \cos \psi_{*}<0$; and for $\varepsilon^{(3)}$, $\sin \psi_{*}<0$.

\section{B. Energy balance approach}

To calculate the threshold amplitude of the parametric field allowing the steady-state oscillating breather to have an arbitrary amplitude, we use the approach proposed in Ref. 13. The method uses energy considerations, and the assumption that the breather mode of the perturbed SG system, $\phi_{\mathrm{br}}(x, t)$, may be chosen in the form of Eq. (4). A similar approach has also been applied to the kink motion in the perturbed SG equation. ${ }^{23-26}$

Let us define the energy of the system as

$$
H \equiv \int_{-\infty}^{\infty}\left(\frac{1}{2} \phi_{x}^{2}+\frac{1}{2} \phi_{t}^{2}+1-\cos \phi\right) d x
$$

For the pure SG breather [Eq. (4)] this energy is $H_{\mathrm{br}}=16 \sqrt{1-\omega_{\mathrm{br}}^{2}}$. In the perturbed SG system Eqs. (16) and (6) yield

$$
\begin{aligned}
\frac{d H}{d t}= & -\alpha \int_{-\infty}^{\infty} \phi_{t}^{2} d x-\beta \int_{-\infty}^{\infty} \phi_{x t}^{2} d x+\varepsilon_{n}^{(1)} \sin \left(\omega t+\theta_{n}^{(1)}\right) \int_{-\infty}^{\infty} \phi_{t} \sin (\phi / n) d x+\varepsilon^{(2)} \sin \left(\omega t+\theta^{(2)}\right) \int_{-\infty}^{\infty} \phi_{t}^{2} d x \\
& +\varepsilon^{(3)} \sin \left(\omega t+\theta^{(3)}\right) \int_{-\infty}^{\infty} \phi_{t} \phi_{t t} d x+\varepsilon^{(4)} \sin \left(\omega t+\theta^{(4)}\right) \int_{-\infty}^{\infty} \phi_{t} \phi_{x x} d x
\end{aligned}
$$

The change in the system energy during one breather period is then found by integrating Eq. (17) over the period 


$$
\Delta H=\int_{0}^{2 \pi / \omega_{\mathrm{br}}} \frac{d H}{d t} d t=I^{\mathrm{in}}-I^{\mathrm{loss}}
$$

where the integrals for the breather energy loss $I^{\text {loss }}$, and the energy input to the breather, $I^{\text {in }}$, must be calculated at $\omega=2 \omega_{\mathrm{br}}$.

$$
I^{\mathrm{loss}}=\alpha \int_{0}^{2 \pi / \omega_{\mathrm{br}}} \int_{-\infty}^{\infty} \phi_{\mathrm{br}, t}^{2} d x d t=32 \pi \alpha \sin ^{-1} \sqrt{1-\omega_{\mathrm{br}}^{2}}=\alpha I^{\text {loss }}(1)
$$

and

$$
I^{\text {in }}=I_{n}^{(1)}+I^{(2)}+I^{(3)}+I^{(4)} .
$$

Here

$$
I_{n}^{(1)}=\varepsilon_{n}^{(1)} I_{n}^{(1)} \cos \theta_{n}^{(1)},
$$

where

$$
\begin{aligned}
& I_{n}^{(1)}(1)=\int_{0}^{2 \pi / \omega_{\mathrm{br}}} \sin \left(2 \omega_{\mathrm{br}} t\right) \int_{-\infty}^{\infty} \phi_{\mathrm{br}, t} \sin \left(\frac{\phi_{\mathrm{br}}}{n}\right) d x d t ; \\
& I^{(2)}=\varepsilon^{(2)} I^{(2)}(1) \sin \theta^{(2)},
\end{aligned}
$$

where

$$
\begin{aligned}
& I^{(2)}(1)=\int_{0}^{2 \pi / \omega_{\mathrm{br}}} \cos \left(2 \omega_{\mathrm{br}} t\right) \int_{-\infty}^{\infty} \phi_{\mathrm{br}, t}^{2} d x d t ; \\
& I^{(3)}=-\varepsilon^{(3)} I^{(3)}(1) \cos \theta^{(3)},
\end{aligned}
$$

where

$$
\begin{aligned}
& I^{(3)}(1)=-\int_{0}^{2 \pi / \omega_{\mathrm{br}}} \sin \left(2 \omega_{\mathrm{br}} t\right) \int_{-\infty}^{\infty} \phi_{\mathrm{br}, t} \phi_{\mathrm{br}, t t} d x d t ; \\
& I^{(4)}=-\varepsilon^{(4)} I^{(4)}(1) \cos \theta^{(3)},
\end{aligned}
$$

where

$$
I^{(4)}(1)=-\int_{0}^{2 \pi / \omega_{\mathrm{br}}} \sin \left(2 \omega_{\mathrm{br}} t\right) \int_{-\infty}^{\infty} \phi_{\mathrm{br}, t} \phi_{\mathrm{br}, x x} d x d t
$$

A power balance is established between energy input and output when the change in the system energy is zero during one breather period. By putting Eq. (18) to zero, and treating the parametric terms independently, we find the balance conditions

$$
\begin{aligned}
& \frac{\varepsilon_{n}^{(1)}}{\alpha} \cos \left(\theta_{n}^{(1)}\right)=\frac{I^{\text {loss }}(1)}{I_{n}^{(1)}(1)}=\frac{\varepsilon_{n, \mathrm{thr}}^{(1)}}{\alpha}, \\
& \frac{\varepsilon^{(2)}}{\alpha} \sin \left(\theta^{(2)}\right)=\frac{I^{\text {loss }}(1)}{I^{(2)}(1)}=\frac{\varepsilon_{\mathrm{thr}}^{(2)}}{\alpha}, \\
& \frac{\varepsilon^{(3)}}{\alpha} \cos \left(\theta^{(3)}\right)=\frac{I^{\text {loss }}(1)}{I^{(3)}(1)}=\frac{\varepsilon_{\mathrm{thr}}^{(3)}}{\alpha}, \\
& \frac{\varepsilon^{(4)}}{\alpha} \cos \left(\theta^{(4)}\right)=\frac{I^{\text {loss }}(1)}{I^{(4)}(1)}=\frac{\varepsilon_{\mathrm{thr}}^{(4)}}{\alpha},
\end{aligned}
$$

where $\varepsilon_{\text {thr }}^{(i)}$ is the threshold value of $\varepsilon^{(i)}$. In Eqs. (25)-(28) the phases $\theta^{(i)}$ are adjusted in order to balance the energy input and output of the driven breather.

We have not been able to calculate any of the integrals [Eqs. (21)-(24)] analytically; however, in the limit $1-\omega_{\mathrm{br}}^{2} \ll 1$, a first-order approximation in $\Omega=1-\omega_{\mathrm{br}}$ yields the results

$$
\begin{aligned}
& \frac{\varepsilon_{n, \mathrm{thr}}^{(1)}}{\alpha} \simeq 2 n\left[1-\Omega \frac{1}{9}\left[7-\frac{16}{n^{2}}-\frac{6}{\pi} \frac{\beta}{\alpha}\right]\right]+O\left(\Omega^{2}\right), \\
& \frac{\varepsilon_{\mathrm{thr}}^{(2)}}{\alpha} \simeq 2\left[1-\Omega \frac{2}{3}\left[1-\frac{1}{\pi} \frac{\beta}{\alpha}\right]\right]+O\left(\Omega^{2}\right), \\
& \frac{\varepsilon_{\mathrm{thr}}^{(3)}}{\alpha} \simeq 2\left[1+\Omega \frac{1}{3}\left[1+\frac{2}{\pi} \frac{\beta}{\alpha}\right]\right]+O\left(\Omega^{2}\right), \\
& \frac{\varepsilon_{\mathrm{thr}}^{(4)}}{\alpha} \simeq 3 \pi \frac{1}{\Omega}+O\left(\Omega^{0}\right),
\end{aligned}
$$

It is important to note that Eq. (29) shows crucial dependence on $n$ when $n$ is compared with the value $\sqrt{16 / 7} \simeq 1.51$. As was found from the NLS treatment in the previous section, we here find that the smallamplitude breather cannot be stabilized by a parametric term $\sim \varepsilon^{(4)}$. Further, we note that if the parametric terms were not considered independently and more than one of the forces were active at a time, we have to make a more careful optimization of the phases. The equations above show that the $\beta$ loss term in Eq. (6) plays a smaller role for the threshold of the driving amplitude than the $\alpha$ loss term for the small-amplitude breather. Hence we will in the following neglect the $\beta$ term in Eq. (6).

\section{Numerical experiments}

In order to check some of our assumptions and our theoretical results we have performed numerical experiments on the system [Eq. (6)] and numerical calculations of the integrals [Eqs. (21)-(24)] together with Eq. (19). Solving Eq. (6) we have used a second-order implicit finite difference method in time and a fourth-order method in space. The space and time grid size were chosen to be $\Delta x=0.02$ and $\Delta t=0.01$ of the normalized units. The breather $\phi(x, t=0)$ is placed at $x=0$ and we have imposed the boundary conditions to be $\phi_{x}(0)=\phi_{x}(L)=0$. The system length was chosen to be $L=25$.

Figure 1 shows our results for $\varepsilon_{n}^{(1)}$. Shown is the perturbation theory results and the results of direct numerical solution of Eq. (6) for $\varepsilon^{(2)}=\varepsilon^{(3)}=\varepsilon^{(4)}=0$, (a) for $\alpha=0.1$ and (b) for $\alpha=0.2$. The solid curves represent the perturbation results [Eq. (25)] with the integral [Eq. (21)] calculated numerically for different values of $n\left(\theta_{n}^{(1)}=0\right)$, the dashed lines show the analytically evaluated asymptotic behavior for $\omega_{\mathrm{br}} \rightarrow 1$ [Eq. (29)]. The error bars represent the direct numerical integration of Eq. (6). The numerical procedure for determining the threshold for stabilization of the breather was the following: for each value of the applied frequency we chose an amplitude of 
the driving field, $\varepsilon$, which can stabilize the breather. Then by decreasing $\varepsilon$ in steps of $\delta \varepsilon$, we monitor for which value of $\varepsilon$ the breather disappears. After each change of $\varepsilon$, we allow the system to relax for 100 periods of the driving field, after which we calculate the total energy of the system. If this energy is within $5 \%$ of the energy of the unperturbed breather [see comment below Eq. (16)] we define the breather to be stabilized, otherwise we define it to be lost. Hence the top of the error bars represents the lowest of the tried values of the amplitude $\varepsilon$ for which the breather was found to be stabilized, and the bottom of the error bars represents the value of $\varepsilon$ for which the breather was lost. As is obvious from the
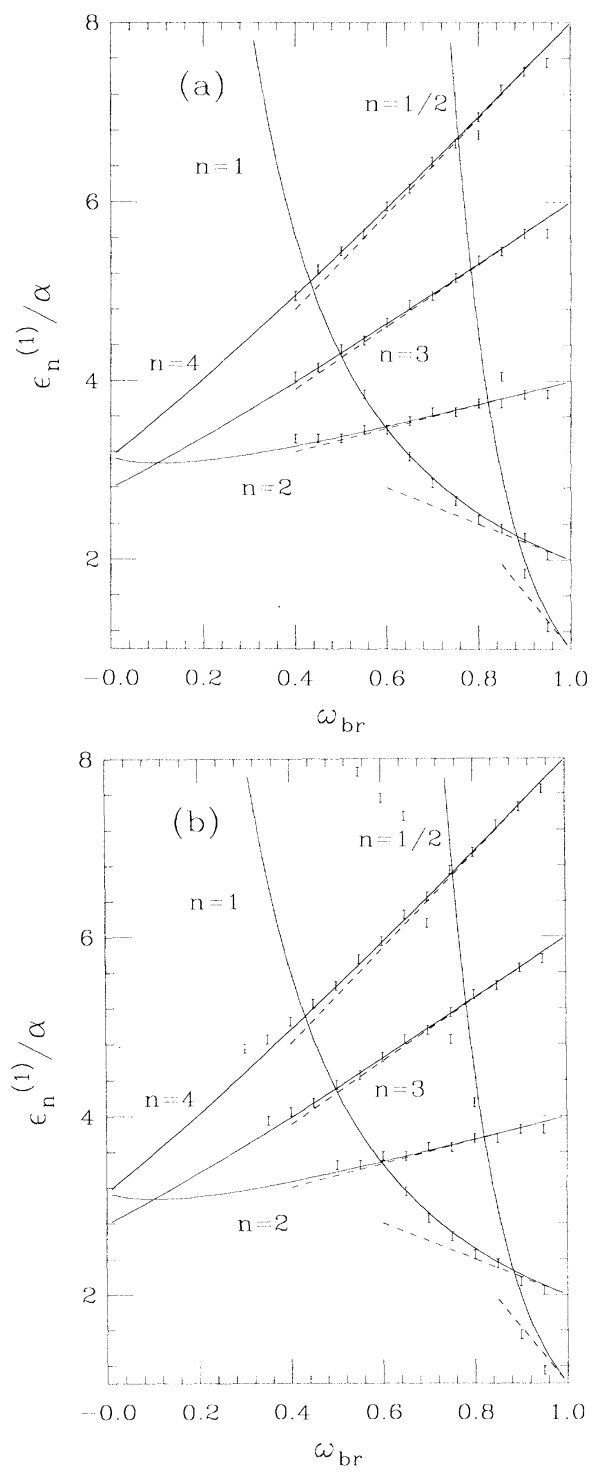

FIG. 1. The threshold value for the amplitude $\varepsilon_{n}^{(1)}$ as a function of the phase-locked breather frequency. Solid lines are the perturbation results using the numerically calculated integral [Eqs. (21) and (19)]. Dashed lines are the analytically calculated tangents for $\omega_{\mathrm{br}} \rightarrow 1$, [Eq. (29)]. Error bars represent the results of numerical simulations on the wave equation (6). Parameters are $L=25$, (a) $\alpha=0.1$, (b) $\alpha=0.2, \beta=0$.

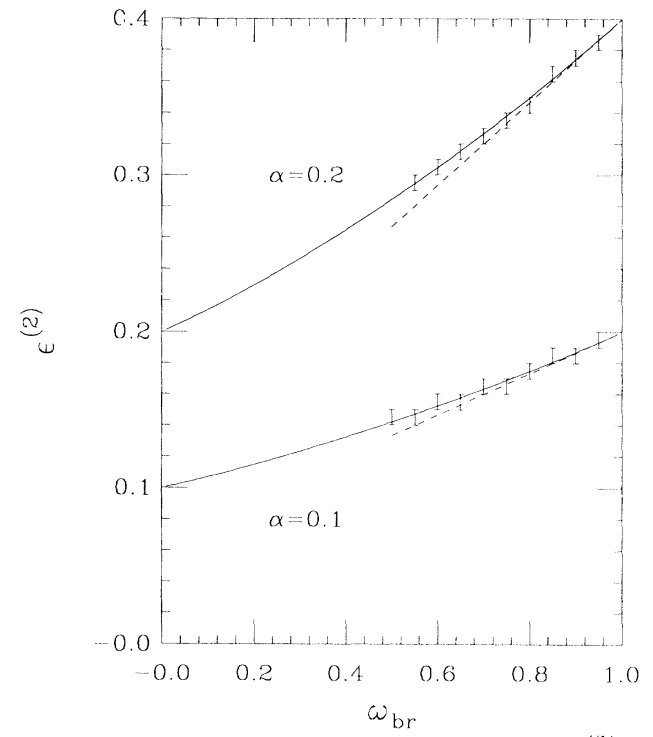

FIG. 2. The threshold value for the amplitude $\varepsilon^{(2)}$ as a function of the phase-locked breather frequency. Solid lines are the perturbation results using the numerically calculated integral [Eqs. (22) and (19)]. Dashed lines are the analytically calculated tangents for $\omega_{\mathrm{br}} \rightarrow 1$, [Eq. (30)]. Error bars represent the results of numerical simulations on the wave equation (6). Parameters are $L=25, \beta=0$.

figures, excellent agreement between the results of numerical experiments and the perturbation results is found.

Figures 2 and 3 show the results for the parametric forces $\varepsilon^{(2)}$ and $\varepsilon^{(3)}$, respectively. Again we find excellent agreement between the results of numerical integration of Eq. (6) and the energy consideration of the threshold of

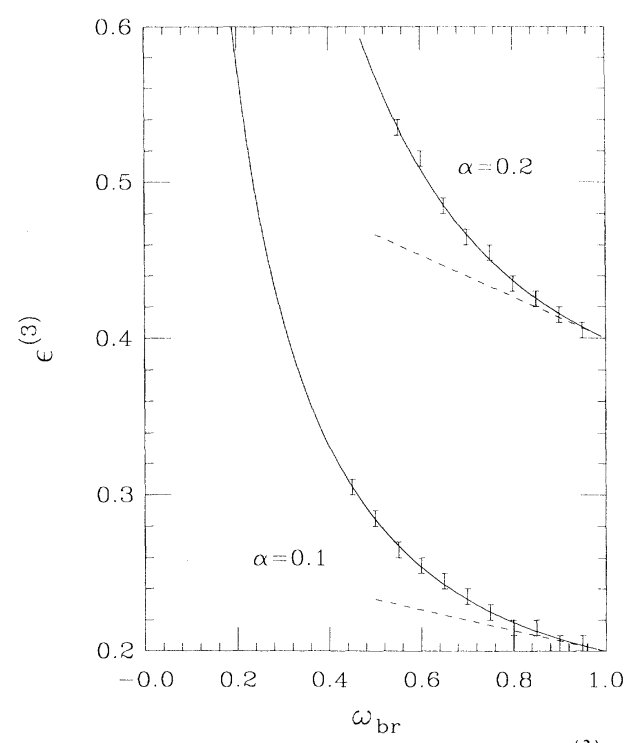

FIG. 3. The threshold value for the amplitude $\varepsilon^{(3)}$ as a function of the phase-locked breather frequency. Solid lines are the perturbation results using the numerically calculated integral [Eqs. (23) and (19)]. Dashed lines are the analytically calculated tangents for $\omega_{\text {br }} \rightarrow 1$, [Eq. (31)]. Error bars represent the results of numerical simulations on the wave equation (6). Parameters are $L=25, \beta=0$. 
the driving amplitude. One thing that is common for all the parametric forces is that they were not able to stabilize the breathers in the low-frequency limit. We have not been able to observe any stabilized breathers for frequencies lower than those indicated by the error bars in Figs. 1-3.

To conclude this section, we note that the energy balance approach to the problem seems to be preferable in comparison to the small-amplitude (NLS) limit. In the latter case, we have only been able to obtain the threshold value in the point $\omega_{\mathrm{br}} \equiv 1$. For this point, we find exact agreement between the NLS treatment and the energy balance approach. However, when $\omega_{\mathrm{br}}=1-\Omega<1$, the energy balance approach, using the numerically calculated integrals [Eqs. (21)-(23)], still gives the correct balance condition while the NLS regime is limited to $\omega_{\mathrm{br}} \equiv 1$. Even the analytically obtained asymptotes [Eqs. $(29)-(31)$ ] show very good agreement in the appropriate limit, $\omega_{\mathrm{br}} \rightarrow 1$.

\section{MODULATION OF THE PHASE-LOCKED BREATHER FREQUENCY}

We have now investigated the possibility of maintaining a phase-locked breather. The threshold value of the amplitude of the applied parametric force was found and the results were verified by numerical experiments. We will now investigate how the phase-locked breather mode responds to a perturbation if the drive amplitude is larger than the threshold value. Following the idea of Ref. 8, we will show that when $\varepsilon_{n}^{(1)}>\varepsilon_{n, \text { thr }}^{(1)}$, we can excite a slow modulation of the locked frequency.

\section{A. Small-amplitude limit}

To analyze the dynamics near the critical point

$$
4 \eta_{*}^{2}=\Omega+\sqrt{\varepsilon_{n}^{2} / 4 n^{2}-\alpha^{2}}, \quad \psi_{*}=\cos ^{-1}\left(2 \alpha n / \varepsilon_{n}\right),
$$

we will derive a system of linear equations describing small modulations of the soliton parameters. Let us put

$$
\eta=\eta_{*}+\delta \eta, \quad \psi=\psi_{*}+\delta \psi,
$$

where $\delta \eta$ and $\delta \psi$ are small deviations from the equilibrium (steady-state) oscillation. Substituting Eq. (33) into Eqs. (11) and (12) and expanding in small $\delta \eta$ and $\delta \psi$, we may derive the set of equations:

$$
\begin{aligned}
& \delta \dot{\eta}=-\eta_{*} \sqrt{\left(\varepsilon_{n}^{(1)} / 2 n\right)^{2}-\alpha^{2}} \delta \psi, \\
& \delta \dot{\psi}=8 \eta_{*} \delta \eta-\alpha \delta \psi .
\end{aligned}
$$

Comparing Eq. (34) with Eq. (35), we may obtain the equation for the phase variation $\delta \psi$,

$$
\delta \ddot{\psi}+\left(\Omega_{M}^{(1)}\right)^{2} \delta \psi=-\alpha \delta \dot{\psi},
$$

where the frequency $\Omega_{M}^{(1)}$ is given by

$$
\left(\Omega_{M}^{(1)}\right)^{2}=\left(\Omega_{0}^{(1)}\right)^{2} \sqrt{\left(\varepsilon_{n}^{(1)} / \varepsilon_{n, \mathrm{thr}}^{(1)}\right)^{2}-1}, \quad\left(\Omega_{0}^{(1)}\right)^{2}=8 \eta_{*}^{2} \alpha .
$$

$\Omega_{M}^{(1)}$ is given by the threshold value of the amplitude of the driving field [Eq. (13)]. An equation similar to Eq.
(36) is valid for the variation of the soliton amplitude $\delta \eta$. Equation (37) describes modulation of the soliton phase (amplitude) with the frequency $\Omega_{M}$; however, because of dissipative losses these modulations are decaying.

Analogous to Eq. (37), we can derive the expressions for the modulation of the breather frequency for the forces $\sim \varepsilon^{(2)}$ and $\sim \varepsilon^{(3)}$. The result is as Eq. (37), but the threshold values for the amplitude of the parametric drive are now given by Eqs. (14) and (15) respectively.

Thus at the level of the small-amplitude approach it has be shown that above the threshold [Eqs. (13)-(15)] the parametrically phase-locked soliton is stable and its parameters are modulated with the frequency [Eq. (37)]. However, this conclusion is valid only when the breather frequency fulfills $1-\omega_{\mathrm{br}}^{2} \ll<1$. In the next subsection we will analyze the more general case.

\section{B. Energy balance approach}

We will here write the unperturbed breather in the form of Eq. (4), where we chose the varying breather frequency in the form

$$
\omega_{\mathrm{br}}=\omega_{0}+\Delta \dot{\theta}, \quad \dot{\omega}_{\mathrm{br}}=\Delta \ddot{\theta},
$$

where $\Delta \theta$ is small and slowly varying and $\omega_{0}$ is the stationary value of $\omega_{\mathrm{br}}$. Throughout this section we will assume that $|\Delta \theta|<<1,|\Delta \dot{\theta}|<<\omega_{0}$. Then the energy change for the breather during one period can be obtained from Eq. (18) as follows: ${ }^{8}$

$$
\Delta H=\frac{2 \pi}{\omega_{\mathrm{br}}} \frac{d H_{\mathrm{br}}}{d t}=-\frac{32 \pi}{\sqrt{1-\omega_{\mathrm{br}}^{2}}} \Delta \ddot{\theta}=I_{n}^{\mathrm{in}}-I^{\text {loss }} .
$$

The loss contribution to the energy change is, of course, given by expressions (19) and (20), but since we here have an extra phase value in consideration, we will modify the expressions by writing $\theta=\theta_{0}+\Delta \theta=\omega_{\mathrm{br}}=\omega_{0}+\Delta \dot{\theta}$ in order to describe the phase mismatch between the breather and the drive as an average phase $\theta_{0}$ and a modulation $\Delta \theta$. During one breather period, we then have the system energy change given by Eq. (18) or (39), provided $|\Delta \theta| \ll<1$. In this equation $I^{\text {loss }}$ is given by Eq. (19). If $\varepsilon_{\text {thr }}$ denotes the threshold value, for which the breather can be maintained (note that we do not specify the type of the driving force at this point), the energy input is given by

$$
I_{n}^{\text {in }}=I^{\text {loss }}\left[1+\Delta \theta \frac{\omega}{\omega_{0}} \frac{\varepsilon}{\varepsilon_{\mathrm{thr}}} \sqrt{1-\left(\varepsilon_{\mathrm{thr}} / \varepsilon\right)^{2}}\right] .
$$

Using this in Eq. (39), we find the general expression

$$
\Delta \ddot{\theta}=-\Omega_{M}^{2} \Delta \theta \text {. }
$$

The result defines the frequency of the breather modulations:

$$
\begin{aligned}
& \Omega_{M}^{2}=\Omega_{0}^{2} \sqrt{\left(\varepsilon / \varepsilon_{\mathrm{thr}}\right)^{2}-1}, \\
& \Omega_{0}^{2}=\alpha\left(\omega / \omega_{0}\right) \sqrt{1-\omega_{0}^{2}} \sin ^{-1} \sqrt{1-\omega_{0}^{2}} .
\end{aligned}
$$

In the small-amplitude limit, when $\sqrt{1-\omega_{0}^{2}}<<1$, Eq. (42) may be transformed into 


$$
\Omega_{M}^{2} \simeq \alpha\left(\omega / \omega_{0}\right)\left(1-\omega_{0}^{2}\right) \sqrt{\left(\varepsilon / \varepsilon_{n, \mathrm{thr}}\right)^{2}-1},
$$

which after substituting $\varepsilon_{n, \mathrm{thr}} \simeq 2 \alpha n, \omega_{0} \equiv \frac{1}{2} \omega$, and $\sqrt{1-\omega_{0}^{2}} \simeq 2 \eta_{*}$, gives exactly the result of Eq. (37) obtained for the NLS equation limit.

In Fig. 4 we have plotted some examples of the time evolution of the breather for different values of the drive amplitude in the case of the drive $\sim \varepsilon^{(2)}$. We show the phase value in the center of the breather, since this quantity very well describes what we want to show here. Clearly, we find that there is a modulation of the breather amplitude (frequency) depending on the amplitude of the drive. In most cases we have observed that the modulation decayed away after some transient time, but in some cases we have not been able to see any decay of the modulation. In these relatively high amplitude drive cases it seems that there exist steady states of modulated breathers. The modulation frequency has been measured for different drive amplitudes and for two values of the driving frequency $(\omega=1.8$ and $\omega=1.9)$. The results of these measurements are shown in Fig. 5, where the solid curve represents the perturbation result, the +'s
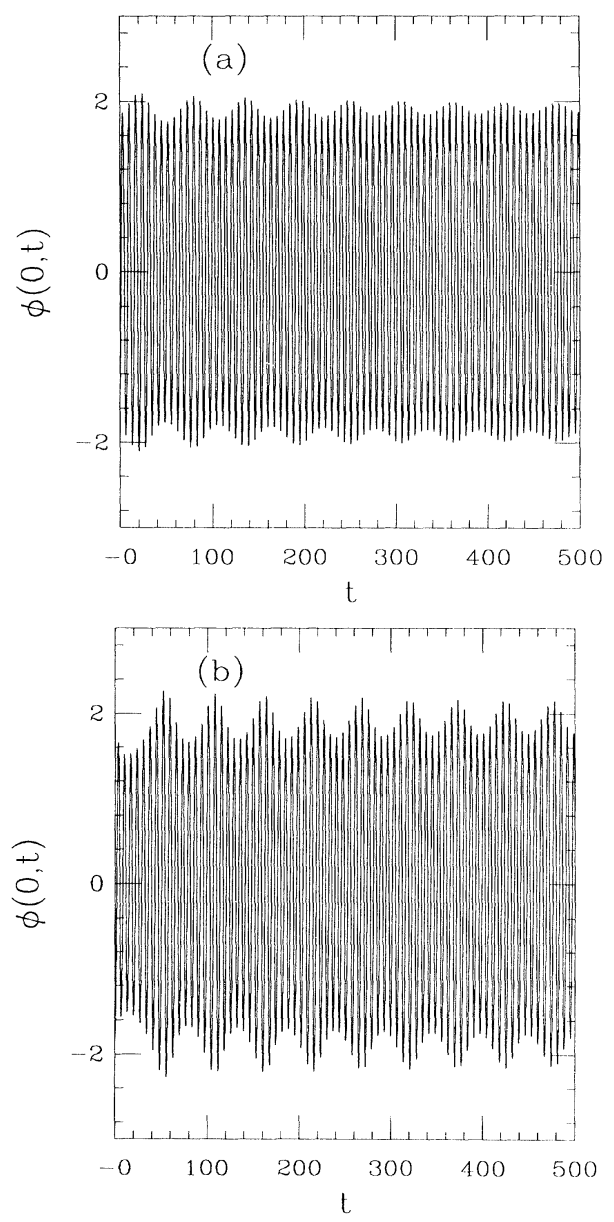

FIG. 4. The time evolution of the center of the breather $\phi(0, t)$ in case of the parametric force $\varepsilon^{(2)}$. Parameters are $\alpha=0.05, \quad \beta=0, \quad \omega_{\mathrm{br}}=0.9, \quad$ and $L=25$. (a) $\varepsilon^{(2)}=0.11$, (b) $\varepsilon^{(2)}=0.12$.

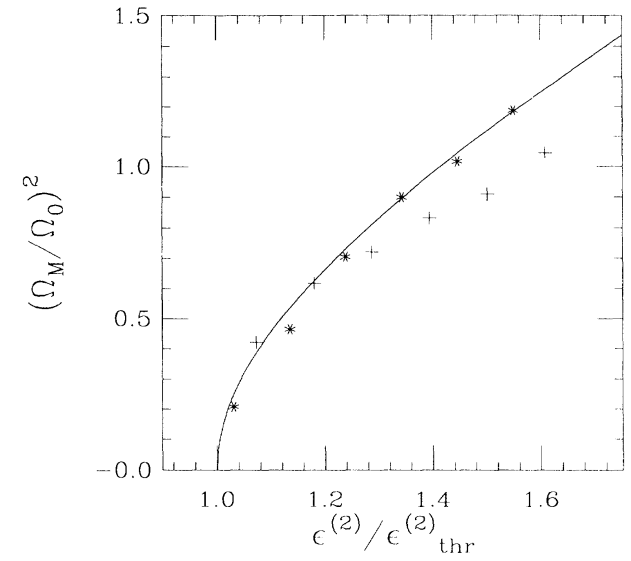

FIG. 5. The square of the modulation frequency as a function of the driving amplitude $\varepsilon^{(2)}$. Parameters are $\alpha=0.05$, $\beta=0$, and $L=25$. ( +) $\omega_{\mathrm{br}}=0.90$. (*) $\omega_{\mathrm{br}}=0.95$. The solid line is the perturbation result [Eq. (42)] and the markers are results of numerical experiments.

represent the $\omega_{0}=0.9$ cases, and the $*$ 's the $\omega_{0}=0.95$ cases. We find very good agreement between the analytical result [Eq. (42)] and the numerical experiments.

From Fig. 4 it is possible to estimate the damping of the modulation. The observed damping is found to be much smaller than what one, a priori, would expect, namely a damping given by $\alpha$, cf. the result of the NLS treatment [Eq. (36)]. In fact, the damping has in most cases been measured to be one or two orders of magnitude smaller than that given by $\alpha$ (see also Ref. 8 ). If the damping is included consistently in the energy balance approach it would yield an additional term,

$$
\frac{\omega_{0}}{\omega} \Omega_{0}^{2} \frac{\partial \ln \varepsilon_{\mathrm{thr}}}{\partial \omega_{0}} \Delta \dot{\theta}=-\alpha^{\prime} \Delta \dot{\theta}
$$

in the right-hand side of Eq. (41). The size of $\alpha^{\prime}$ is one to two orders of magnitude smaller than $\alpha$ in most cases, but the sign is given by the sign of $-\left(\partial \varepsilon_{\mathrm{thr}}\right) /\left(\partial \omega_{0}\right)$, which in some of the cases is negative, as seen in Figs. 1-3. In a perturbation treatment like this, the correct conclusion is that the damping is very small and we are not able to derive any details. This is because the effective damping of the modulation arises as a small difference between two almost equal numbers, $\sim \alpha$ and $\sim \varepsilon$.

Let us as a final comment in this section note that the derivation of the expression for the modulation frequency has been done without any specification of the nature of the driving force. This indicates that the expression may be applicable for a general type of force. As an example, we note that the expression for the modulation frequencies in the case of an ac driving force ${ }^{8}$ is completely identical to our Eq. (37) when $\omega \equiv \omega_{0}$, i.e., when the frequency of the driving term equals the frequency of the stabilized breather. In Ref. 8 it was shown that there was good agreement between the analytical expression for the modulation and the numerical experiments. Here, we will try yet another different driving mechanism to see if the stabilized breather will be modulated in frequency according to Eq. (37). In Ref. 27 it was demonstrated that a 


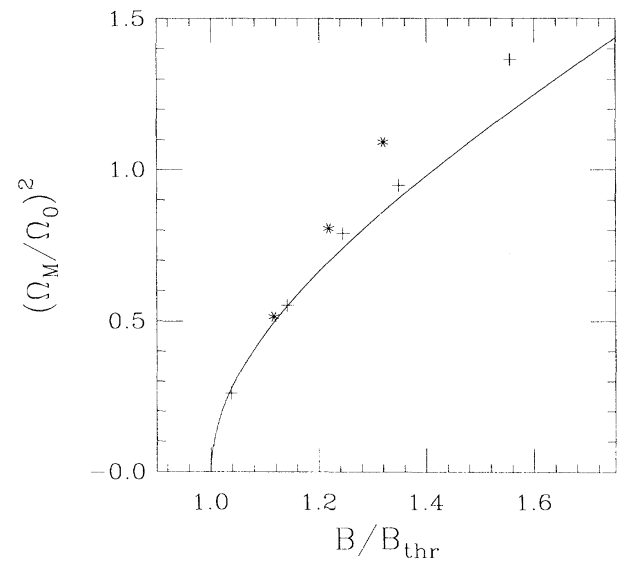

FIG. 6. The square of the modulation frequency as a function of the amplitude of the point drive $B$. Parameters are $\alpha=0.025, \beta=0$, and $L=25$. $\left(^{*}\right) \omega_{\mathrm{br}}=0.90$. (+) $\omega_{\mathrm{br}}=0.80$. The solid line is the perturbation result [Eq. (42)] and the markers are results of numerical experiments.

breather could be stabilized in a system defined by Eq. (6) for $\varepsilon^{(i)}=\beta=0$ if the following boundary conditions are imposed:

$$
\phi_{x}(0, t)=B \cos \omega_{0} t, \quad \phi_{x}(L, t)=0,
$$

and the threshold value for the driving amplitude was found to be

$$
B_{\mathrm{thr}}=2 \alpha \sqrt{\left(1+\omega_{0}\right) /\left(1-\omega_{0}\right)} \sin ^{-1} \sqrt{1-\omega_{0}^{2}} .
$$

Using this threshold value in Eq. (42) for $\Omega_{0}^{2}$ and comparing the result to the results of numerical simulations of the system defind above, we found good agreement as shown in Fig. 6. Here, we have shown the general perturbation result as a solid curve and the results of the numerical experiments as markers for two different values of the driving frequency.

\section{CONCLUSION}

In conclusion, we have analyzed the breather dynamics in the perturbed sine-Gordon system, driven by different types of parametric forces. The condition for stabilization of the breathers has been given using two different perturbation approaches. One of the methods, valid only in the small-amplitude limit (the NLS regime), gives the correct threshold value for the amplitude of the driving force in the point where $\omega_{\mathrm{br}} \equiv 1$. The other approach, the energy balance approach, gives the threshold value for any value of the breather frequency, $-1<\omega_{\mathrm{br}} \leq 1$. Comparisons between the obtained perturbation results and results of numerical experiments made on the perturbed sine-Gordon equation show almost perfect agreement for the energy balance approach.

In the second part of the paper, we have demonstrated that a breather, out of the phase-locked equilibrium, will relax with a slow oscillating modulation around the frequency and amplitude of the phase-locked state. This modulation frequency has been found analytically for a general type of driving force and it has been compared to results of numerical experiments for different types of external drives. It is interesting to note that the modulation frequency is, independently of the type of external force, directly given by the damping coefficient $\alpha$ in the field equation.

\section{ACKNOWLEDGMENTS}

Yuri Kivshar thanks the Physics Laboratory I at the Technical University of Denmark for kind hospitality during his visit. $\mathrm{He}$ is also indebted to Igor Barashenkov for useful remarks. The work of Yuri Kivshar was partially supported by the University Complutense through the Programma Sabaticos de Complutense. Niels Grønbech-Jensen is grateful to the Carlsberg Fondet for financial support during the final part of this work.
*On leave from Institute for Low Temperature Physics and Engineering, 47 Lenin Avenue, 310164 Kharkov, Ukraine.

${ }^{1}$ Yu. S. Kivshar and B. A. Malomed, Rev. Mod. Phys. 61, 763 (1989).

${ }^{2}$ D. J. Kaup and A. C. Newell, Phys. Rev. B 18, 5162 (1978).

${ }^{3}$ A. R. Bishop, K. Fesser, P. S. Lomdahl, and S. E. Trullinger, Physica D 7, 259 (1983).

${ }^{4}$ K. Nozaki and N. Bekki, J. Phys. Soc. Jpn. 54, 2363 (1985).

${ }^{5}$ A. Mazor, A. R. Bishop, and D. W. McLaughlin, Phys. Lett. A 119, 273 (1986)

${ }^{6}$ P. S. Lomdahl and M. R. Samuelsen, Phys. Rev. A 34, 664 (1986).

${ }^{7}$ A. Mazor and A. R. Bishop, Physica D 27, 269 (1987).

${ }^{8}$ P. S. Lomdahl and M. R. Samuelsen, Phys. Lett. A 128, 427 (1988).

${ }^{9}$ M. Taki, K. M. Spatschek, J. C. Fernandez, R. Grauer, and G. Reinisch, Physica D 40, 65 (1989).

${ }^{10}$ Niels Grønbech-Jensen, Boris A. Malomed, and Mogens R. Samuelsen, Phys. Lett. A 166, 347 (1992).

${ }^{11}$ V. E. Zakharov, V. S. L'vov, and S. S. Starobinetz, Usp. Fiz. Nauk. 114, 609 (1974) [Sov. Phys. Usp. 17, 896 (1975)].
${ }^{12}$ M. M. Bogdan, A. M. Kosevich, and I. V. Manzkos, Fiz. Nizk. Temp. 11, 991 (1985) [Sov. J. Low Temp. Phys. 11, 547 (1985)].

${ }^{13}$ N. Grønbech-Jensen, Yu. S. Kivshar, and M. R. Samuelsen, Phys. Rev. B 43, 5698 (1991).

${ }^{14}$ I. V. Barashenkov, M. M. Bogdan, and V. I. Kozobov, Europhys. Lett. 15, 113 (1991).

${ }^{15}$ C. Vanneste, A. Gilabert, P. Sibillot, and D. B. Ostrowsky, J. Low Temp. Phys. 45, 517 (1981).

${ }^{16}$ R. Sobolewski and C. V. Stancampiano, Phys. Rev. A 31, 6063 (1985).

${ }^{17}$ G. Cicogna and L. Frinzoni, Phys. Rev. A 42, 1901 (1990).

${ }^{18}$ H. Mikeska, J. Phys. C 11, 129 (1978).

${ }^{19}$ V. G. Bar'yakhtar, B. A. Ivanov, and A. A. Sukstansky, Zh. Eksp. Teor. Fiz. 78, 1509 (1980) [Sov. Phys. JETP 51, 757 (1980)].

${ }^{20}$ H. T. J. Smith and J. A. Blackburn, Phys. Rev. A 43, 5698 (1989).

${ }^{21}$ D. W. McLaughlin and A. C. Scott, Phys. Rev. A 18, 1652 (1978).

${ }^{22}$ Niels Grønbech-Jensen, Ph.D. thesis, The Technical Universi- 
ty of Denmark, 1991 (unpublished).

${ }^{23}$ O. H. Olsen and M. R. Samuelsen, Phys. Rev. B 28, 210 (1983).

${ }^{24}$ N. Grønbech-Jensen, P. S. Lomdahl, and M. R. Samuelsen, Phys. Lett. A 154, 14 (1991).

${ }^{25}$ Niels Grønbech-Jensen, Phys. Rev. B 45, 7315 (1992).
${ }^{26}$ Niels Grøbech-Jensen, Boris A. Malomed, and Mogens R. Samuelsen, Phys. Rev. B 46, 294 (1992).

${ }^{27}$ O. H. Olsen and M. R. Samuelsen, Appl. Phys. Lett. 47, 1007 (1985); Phys. Rev. B 34, 3510 (1986); Phys. Lett. A 119, 391 (1987). 